\title{
G sememe \\ Clinical Characteristics of Nodular Fasciitis of Ear in Children
}

\section{Xiaoxu Wang}

National Center for Children's Health: Beijing Children's Hospital Capital Medical University

Wei Liu

National Center for Children's Health: Beijing Children's Hospital Capital Medical University

\section{Lejian He}

National Center for Children's Health: Beijing Children's Hospital Capital Medical University

Min Chen

National Center for Children's Health: Beijing Children's Hospital Capital Medical University

Jianbo Shao

National Center for Children's Health: Beijing Children's Hospital Capital Medical University

Xiao Zhang

National Center for Children's Health: Beijing Children's Hospital Capital Medical University

Ning Ma

National Center for Children's Health: Beijing Children's Hospital Capital Medical University

Yanhong Li

National Center for Children's Health: Beijing Children's Hospital Capital Medical University

Jie Zhang ( $\nabla$ stzhangj@263.net)

Beijing Children's Hospital, Capital Medical University https://orcid.org/0000-0002-1621-7779

\section{Research Article}

Keywords: Nodular fasciitis, Children, Ear, Manifestations, USP6 gene

Posted Date: May 10th, 2021

DOl: https://doi.org/10.21203/rs.3.rs-484827/v1

License: (c) (1) This work is licensed under a Creative Commons Attribution 4.0 International License.

Read Full License

Version of Record: A version of this preprint was published at European Journal of Pediatrics on October 11th, 2021. See the published version at https://doi.org/10.1007/s00431-021-04274-x. 


\section{Abstract}

Purpose Summarized the clinical characteristics and diagnosis and treatment process of three cases of nodular fasciitis of ear, to provide a basis for clinical diagnosis and treatment.

Methods Reviewed the clinical manifestations, images, pathology, treatment and postoperative follow up results of three cases of pediatric nodular fasciitis in the Department of Otorhinolaryngology, Head and Neck Surgery, Beijing Children's Hospital, Capital Medical University from 2018 to 2020.

Results The average age at diagnosis were 24 months, with two girls and a boy. Two lesions were found in the left ear and one in the right ear. All cases had a history of biopsy before surgery. Two of three cases showed a sign of rapid growth after biopsy and three of which were ineffective in anti-inflammatory treatment. FISH test for USP6 were performed in two of the three cases with positive results. Three lesions show a hypointensity or isointensity on T1-weighted MRI and a heterogeneous hyperintensity on T2-weighted MRI. "Fascial tail” sign was found on image of all three cases. All lesions underwent surgical resection. Follow-up showed no recurrence and had an intact ear appearance.

Conclusion The early misdiagnosis rate of nodular fasciitis of the ear is high. Combine clinical features with imaging findings may improve the accuracy of preoperative diagnosis. Besides the appearance of pathology, USP6 gene test is also an important tool in the diagnosis. The final diagnosis should be based on comprehensive assessment. Complete surgical resection can prevent recurrence.

\section{What Is Known}

- Nodular fasciitis is a rare benign, self-limiting, reactive soft tissue tumor which is composed of fibroblasts and myofibroblasts.

- Nodular fasciitis can occur anywhere but is is rare around the ear particularly in children.

\section{What is New}

- Combined with the imaging manifestations, the specificity of genes and biopsy can improve the diagnostic rate.

- The treatment of ear nodular fasciitis is not only expected to be self-limited, but also has a satisfactory prognosis after surgical resection on the premise of weighing the reconstruction of appearance.

\section{Introduction}

Nodular fasciitis (NF) is a rare benign, self-limiting, reactive soft tissue tumor which is composed of fibroblasts and myofibroblasts. This disease was first described by Konwaler in 1955, when he called it pseudosarcomatous fasciitis[1]. The disease can be misdiagnosed as a malignant lesion because of its rapid growth and high cellularity upon histopathological examination. This lesion can occur anywhere, 
mostly in the upper limb (48\%), followed by the trunk (20\%) and the head and neck (13-20\%) $[2,3]$. The age of onset is usually between 20 and 40 years old, and the prevalence rate in children is about $10 \%[4$, 5]. NF that occurs around the ear is rare particularly in children. To the best of our knowledge, only few cases of NF of the ear have been reported[6,7], and thus, there is a limited description of the associated findings. In order to strengthen our diagnostic awareness of pediatric NF around the ear, we retrospectively analyzed the clinical features, diagnosis and treatment of three cases in our department.

\section{Materials And Methods}

This study has been approved by the ethics review committee of our institution. We conducted a retrospective analysis of data collected from three children with NF who were treated in the Department of Otolaryngology of Beijing Children's Hospital, Capital Medical University. Combined manifestations, cytomorphologic and immunohistochemical features confirmed the diagnosis of NF.

All the three patients underwent computer tomography (CT), magnetic resonance imaging (MRI) and ultrasonography (US). Case 1 only underwent regular MR images and CT scan and case2 and case3 had both regular and enhanced MR images and CT scan. Imaging features of the lesions were characterized according to the size, margin, signal intensity and degree of enhancement. The density and signal intensity were documented as hypo-, iso-, or hyper-, respectively, relative to that of muscle. The reviewed of the imaging (CT/MRI) was completed by four senior otolaryngologists. The pathological sections underwent hematoxylin and eosin (H\&E) staining. The immunohistochemical staining (IHC) results of smooth muscle actin (SMA), catenin, Desmin, S-100, CD34 and CD68 were all documented. Fluorescence in situ hybridization (FISH) for the ubiquitin-specific protease 6 (USP6) rearrangement was performed and the probe was USP6 gene two-color separation probe.

\section{Results}

\section{Case 1}

A three-year-old girl presented a painless mass in front of the right ear that has been present for one and a half years. (Fig1) Regular anti-infection treatment was not effective. The mass was biopsied before surgery, histology revealed the diagnosis of NF. Temporal bone CT showed an irregular soft tissue mass with uniform density in the anterior and inferior part of the right external auditory canal. On MR image, the mass showed hypointense on T1-weighted imagine (T1WI) signal related to skeletal muscle. Hyperintense signals were showed on T2-weighted imagine (T2WI) and STIR sequences. US exam showed a $41 \times 21 \times 33 \mathrm{~mm}$ ovoid hypoechoic nodule with distinct margins.

The child underwent surgery under general anesthesia. We made a longitudinal incision along the preauricular bulge. Then cut the subcutaneous tissue, and exposed the mass. The border of the mass was obvious. After completely resected, the specimen was sent to pathology. Histopathologically, the lesion was compatible with a diagnosis of NF. IHC staining showed positivity for SMA and catenin, CD68 
was focally positive, Ki-67 was $10 \%$ positive and CD34, S-100 and Desmin were negative. There was no recurrence 31 months after operation by MR image.

\section{Case 2}

A one year and five months boy was admitted to our department with a mass in his right pinna during four months(Fig2). He went through two surgeries to cut off the mass before this admission. Besides, the mass caused no pain before surgery. The pathology showed myofibroblastic tumor. After the second surgery the wound did not heal. Then the mass reappeared and grew rapidly. Anti-infection treatment was administered during this period but it doesn't work. The boy underwent MR image and US in our department after this admission. MRI demonstrated a nodule with an isointense on T1WI and an hyperintense on T2WI and STIR sequences. Enhanced MRI showed inhomogeneous enhancement at the edge of the lesion and no enhancement in the interior of the lesion. US examination revealed a solid mass of irregular hypoechoic, which had an un-even internal echo. The mass was about $30 \times 18 \times 28 \mathrm{~mm}$ in size.

The operative incision made from retroauricular sulcus. During the operation, it was seen that the lesion did not have a complete capsule with a rotten fish-like appearance and crisp texture. The mass did not penetrate the fascia layer behind the ear, but invaded the cartilage of the cavum concha forward. The incisal margins were sent to frozen pathology for several times until the incisal margins were negative. Triamcinolone acetonide was injected to the operation cavity at the end of the operation. The postoperative pathology confirmed the diagnosis of NF. IHC staining showed positivity for SMA, CD68 was focally positive, Ki-67 was $15 \%$ positive and CD34, Desmin, and S-100 were negative. FISH test result showed that $34 \%$ of 100 cells showed USP6 gene rearrangement. At present, it recovered well with nine months after operation.

\section{Case 3}

A one year and seven months girl with a painless and progressive mass at the external orifice of the left ear during six months(Fig3). And the mass broke and bled in the last month. Then a biopsy was performed in another hospital, and the pathological result was inflammatory granuloma. The mass showed a sign of enlargement after the biopsy. Temporal bone CT showed that an oval low-density tissue in the left auricle with surrounding soft tissue thickening. MR image features include hypointense relative to skeletal muscle on T1WI and Hyperintense on T2WI or STIR sequences with Inhomogeneous enhancement after contrast injection. The whole lesion was about $17 \times 16 \times 9 \mathrm{~mm}$ in US. In addition, the lesion did not show any intrinsic vascularity, though the surrounding soft tissue exhibited increased vascularity on doppler US examination.

We made a surgical incision along the edge of the mass. In the operation found that the tumor did not have a complete capsule and the texture is solid. The incisal margins were sent to frozen pathology, and the lesion was completely resected. Finally, the skin of the mastoid area was taken as a free skin flap and transplanted into the skin defect of the ear canal. IHC staining showed positivity for SMA and CD68, Ki-67 was $10 \%$ positive, and CD34, S-100, and Desmin were negative. The diagnosis of NF was made. FISH test 
result showed that $33 \%$ of 100 cells showed USP6 gene rearrangement. At present, it recovered well eight months after operation.

Table1 summarizes the clinical characteristics and examination data of the three children. The average age of onset was 14 months, with two girls and one boy. The maximum diameters of three lesions were $2.9 \mathrm{~cm}, 2.8 \mathrm{~cm}, 1.7 \mathrm{~cm}$, respectively. All of the three children had no history of trauma. The antiinflammatory treatment was ineffective in all three patients. Case 1 and case 3 had a history of biopsy, and case 2 had a surgical history of mass resection. These three masses were all surgically removed finally in our hospital. Regular follow-up was carried out after the operation and none of the three cases recurrence. They all have intact ear appearance.

\section{Discussion}

$\mathrm{NF}$ is a relatively common mass-forming and self-limited subcutaneous pseudosarcomatous myofibroblastic proliferation. In the 2013 WHO soft tissue tumor classification, it is classified as fibroblast / myofibroblastoma[8]. Pediatric NF around the ear is rare. Thompson et al. [9]reported the largest number of cases of external ear NF in 2001. The mean age, 27.4 years(1-74). The author did not provide further age details. Through literature search, it is also found that most of the previous reports are case reports $[6,7,10]$. We are the only report that had 3 cases of NF of the ear in children with complete medical history and prognosis.

Ear NF in children is difficult to distinguish from other soft tissue lesions in appearance. It is also easy to be misdiagnosed as other infectious mass which has a higher incidence in children clinically. And all three patients had a misdiagnosis experience and experienced ineffective antibiotic treatment.

The cause of the disease is unclear. Previously, it was thought to be related to local trauma. In 2011, Erickson-Johnson et al reported the mechanism of NF seems to involve USP6 transcriptional upregulation due to its high level of the fusion with MYH9.[11] It provided evidence for clonal neoplastic origin of NF. Further investigations in this filed are needed. In our study, all of the three children had no history of trauma. But there was a sign of rapid enlargement after biopsy in case 3 and a sign of reappeared and grew rapidly after surgical resection in case 2 . We considered the possibility that external stimuli might cause enlargement of the existing mass.

Radiologically, MR imaging of the ear can better evaluate the size, extent, and the nature of the mass than CT. Most of the T1-weighted MRI classically shows hypointensity or isointensity to adjacent skeletal muscles and T2-weighted imaging or STIR weighted sequences reveals heterogeneous hyperintensity.[12, 13] In this study, the MRI signal characteristics of the lesions were similar to those of previous studies. In addition, the" fascial tail" sign is valuable to suggest the diagnosis of NF.[14] All of the three children were found with the "fascial tail" sign characterized as thickening of adjacent fascial layer with obvious enhancement in the shape of linear or rat tail on MR image. The fascia tail sign is not a specific sign of $\mathrm{NF}$, and some malignant soft tissue tumors also have a tail sign.[15] However, the relatively specific 
clinical manifestations of NF, combined with the fascia tail sign on MRI images, can make a consideration of NF.

Histologically, NF shows proliferative, tumourlike, immature fibroblastic lesions with rich cellularity and mitotic activity. Due to these features, NF is often misdiagnosed as a sarcoma. Furthermore, EricksonJohnson et al.[11] First reported USP6 gene rearrangement on chromosome 17p13 as a specific finding in NF in 2011. They also reported a sensitivity of $93 \%$ and a specificity of $100 \%$ for USP6 rearrangements as a diagnostic marker for NF as confirmed by FISH. The result considered positive if $>10 \%$ of 200 cells showed split signals[11, 16]. At present, USP6 rearrangements and USP6 fusion genes can be used as a diagnostic tool for NF. In our cases, two of three cases were positive for the rearrangement of USP6 gene and one case didn't perform this test.

Treatment options for NF include observation and surgically resection. Local resection is the effective treatment for this lesion. The recurrence rate of Local resection is reported to be $1-2 \%[17]$. According to the research of Thompson et al.[9], NF has a higher propensity for local recurrence in the auricular area(9.3\%). They explained that this resulted from increased auricular trauma and the difficulty in complete excision due to the anatomical position. Our three children were already followed up for 31,9 , and 8 months, and there no recurrence was found. In some case reports, regression can be observed after a fine needle aspiration biopsy[18]. Even without excision, spontaneous regression also has been observed.[19] We recommend conservative observation in the condition that function and appearance were well. Once the deeper tissue is invaded, it should be completely surgically removed.

\section{Conclusion}

NF should be considered in the differential diagnosis of young children presenting with rapidly enlarging painless masses around the ear and failing to respond to anti-infective treatment. The combination of clinical manifestations with imaging and preoperative biopsy can reduce the misdiagnosis rate. For ear $\mathrm{NF}$, the significance of local appearance preservation or reconstruction is higher than lesions of other position, so it is necessary to take into account the prognosis and beauty in the choice of treatment. Local complete resection can be cured. However, those small cases that do have not invade the surrounding structure can observe several weeks.

\section{Declarations}

\section{Acknowledgments}

The authors would like to thank colleagues, patients, and their families for their support for this study. We wish to acknowledge the funding support from the Special Fund of the Pediatric Medical Coordinated Development Center of Beijing Hospitals Authority and Beijing Hospitals Authority "Ascent Plan" .

Funding Partial financial support was received from the Special Fund of the Pediatric Medical Coordinated Development Center of Beijing Hospitals Authority (No. XTYB201828) and Beijing Hospitals 
Authority “Ascent Plan” (No. DFL20191201).

Conflict of interests The authors have no financial or proprietary interests in any material discussed in this article.

Availability of data and material All data and materials are transparent.

\section{Code availability N/A}

Authors' contributions All authors contributed to the study conception and design. Material preparation, data collection and analysis were performed by Jie Zhang, Xiaoxu Wang, Wei Liu, Lejian He, Min Chen, Jianbo Shao, Xiao Zhang, Ning Ma, Yanhong Li. The first draft of the manuscript was written by Xiaoxu Wang and all authors commented on previous versions of the manuscript. All authors read and approved the final manuscript.

Ethics approval Ethical approval was waived by the local Ethics Beijing Children's Hospital, Capital Medical University in view of the retrospective nature of the study and all the procedures being performed were part of the routine care.

Consent to participate Informed consent was obtained from all individual participants included in the study.

Consent for publication The participant has consented to the submission of the original article to the journal.

\section{References}

1. Konwaler BE, Keasbey L, Kaplan L. Subcutaneous pseudosarcomatous fibromatosis (fasciitis)[J]. Am J Clin Pathol, 1955, 25(3): 241-252. https://doi.org/10.1093/ajcp/25.3.241

2. Shimizu S, Hashimoto H, Enjoji M. Nodular fasciitis: An analysis of 250 patients[J]. Pathology, 1984, 16(2): 161-166. https://doi.org/10.3109/00313028409059097

3. Montgomery EA, Meis JM. Nodular fasciitis. Its morphologic spectrum and immunohistochemical profile[J]. Am J Surg Pathol, 1991, 15(10): 942-948.

4. Hseu A, Watters K, Perez-Atayde A, et al. Pediatric nodular fasciitis in the head and neck: Evaluation and management[J]. JAMA Otolaryngol Head Neck Surg, 2015, 141(1): 54-59. https://doi.org/10.1001/jamaoto.2014.2797

5. Stout AP. Pseudosarcomatous fascitis in children[J]. Cancer, 1961, 14: 1216-1222. https://doi.org/10.1002/1097-0142(196111/12)14:6<1216::aid-cncr2820140611>3.0.co;2-w

6. Al Rahbi M, Al-Kindi H, Al-Sheibani S. Post-aural nodular fasciitis[J]. Oman Med J, 2015, 30(1): 63-65. https://doi.org/10.5001/omj.2015.12 
7. Jovanovic MB, Cvorovic L, Milosevic S, et al. Unusual presentation of nodular fasciitis as a rapidly growing haemorrhagic temporal mass[J]. J Craniomaxillofac Surg, 2012, 40(1): 33-35. https://doi.org/10.1016/j.jcms.2011.02.002

8. Jo VY, Fletcher CD. Who classification of soft tissue tumours: An update based on the 2013 (4th) edition[J]. Pathology, 2014, 46(2): 95-104. https://doi.org/10.1097/pat.0000000000000050

9. Thompson LD, Fanburg-Smith JC, Wenig BM. Nodular fasciitis of the external ear region: A clinicopathologic study of 50 cases[J]. Ann Diagn Pathol, 2001, 5(4): 191-198. https://doi.org/10.1053/adpa.2001.26969

10. Pandian TK, Zeidan MM, Ibrahim KA, et al. Nodular fasciitis in the pediatric population: A single center experience[J]. J Pediatr Surg, 2013, 48(7): 1486-1489. https://doi.org/10.1016/j.jpedsurg.2012.12.041

11. Erickson-Johnson MR, Chou MM, Evers BR, et al. Nodular fasciitis: A novel model of transient neoplasia induced by myh9-usp6 gene fusion[J]. Lab Invest, 2011, 91(10): 1427-1433. https://doi.org/10.1038/labinvest.2011.118

12. Khuu A, Yablon CM, Jacobson JA, et al. Nodular fasciitis: Characteristic imaging features on sonography and magnetic resonance imaging[J]. J Ultrasound Med, 2014, 33(4): 565-573. https://doi.org/10.7863/ultra.33.4.565

13. Kim ST, Kim HJ, Park SW, et al. Nodular fasciitis in the head and neck: Ct and mr imaging findings[J]. AJNR Am J Neuroradiol, 2005, 26(10): 2617-2623.

14. Hu PA, Zhou ZR. Imaging findings of radiologically misdiagnosed nodular fasciitis[J]. Acta Radiol, 2019, 60(5): 663-669. https://doi.org/10.1177/0284185118788894

15. Yoo HJ, Hong SH, Kang Y, et al. Mr imaging of myxofibrosarcoma and undifferentiated sarcoma with emphasis on tail sign; diagnostic and prognostic value[J]. Eur Radiol, 2014, 24(8): 1749-1757. https://doi.org/10.1007/s00330-014-3181-2

16. Oliveira AM, Chou MM. Usp6-induced neoplasms: The biologic spectrum of aneurysmal bone cyst and nodular fasciitis[J]. Hum Pathol, 2014, 45(1): 1-11.

https://doi.org/10.1016/j.humpath.2013.03.005

17. Enzinger FM, Weiss SW: Soft Tissue Tumors, 2nd ed, pp 103-112, CV Mosby, St. Louis, 1988[M].

18. Wong NL, Di F. Pseudosarcomatous fasciitis and myositis: Diagnosis by fine-needle aspiration cytology[J]. Am J Clin Pathol, 2009, 132(6): 857-865. https://doi.org/10.1309/ajcpleps44pjhdpp

19. Hüter EN, Lee CC, Sherry RM, et al. Spontaneous regression and recurrence in a case of nodular fasciitis[J]. Acta Derm Venereol, 2009, 89(4): 438-439. https://doi.org/10.2340/00015555-0664

\section{Tables}

Table 1. Clinical characteristics of three cases. 


\begin{tabular}{|llll|}
\hline & Case 1 & Case 2 & Case 3 \\
\hline Age ${ }^{1}$, month/Sex & $36 / \mathrm{G}$ & $17 / \mathrm{B}$ & $19 / \mathrm{G}$ \\
\hline Side & Right & Right & Left \\
\hline Location & Preauricular & Pinna & External auditory meatus \\
\hline Diameter ${ }^{2}$, cm & 2.9 & 2.8 & 1.7 \\
\hline Course, month & 18 & 4 & 6 \\
\hline Trauma history & without & without & without \\
\hline $\begin{array}{l}\text { Clinical features of the } \\
\text { mass }\end{array}$ & Stiff, & Stiff, painless and rapid & Stiff, painless and rapid \\
\hline Plain/Enhanced CT scan & Yes/No & growth & growth \\
\hline $\begin{array}{l}\text { Regular/Enhanced MR } \\
\text { scan }\end{array}$ & Yes/No & Yes/Yes & Yes/Yes (Other hospital) \\
\hline Sonography & Yes & Yes & Yes/Yes \\
\hline $\begin{array}{l}\text { FlSH for USP6 } \\
\text { Subtype }\end{array}$ & No & Yes & Yes \\
\hline $\begin{array}{l}\text { Follow-up, } \\
\text { month/Recurrence }\end{array}$ & Fascial & Subcutaneous & Yes \\
\hline
\end{tabular}

1 Age at diagnosed.

2 Maximum diameter on MR image.

3 Clinical features of the mass first found.

\section{Figures}



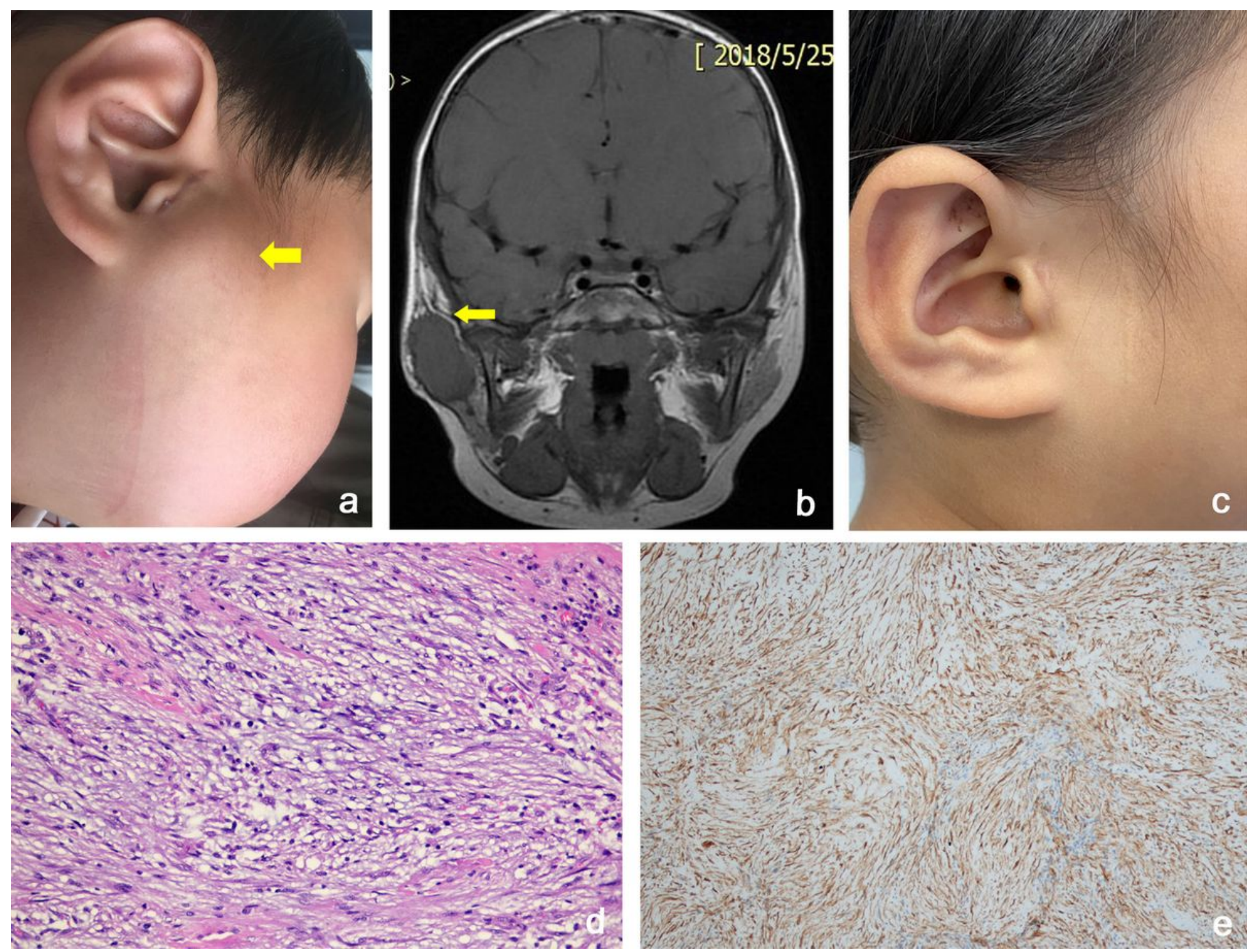

Figure 1

$a$ is Pre-operative clinical photograph of case 1 which shows a mass in front of the right ear. Fig1-b shows Coronal T1 weighted MR image of case 1. An ovoid isointense mass in the temporal, which showed hypointense on T1W1. Three is a "fascial tail" sign (arrow) in image. Fig1-c is a photograph of ear in 27 months after operation. There is an intact ear appearance without recurrence and other postoperative complications. NF was composed of spindle cells with vascular proliferation and lymphocytic infiltration in a myxoid stroma (Fig1-d: H\&E stain, $\times 200$ ). IHC staining showed positivity for SMA (Fig1-e) 

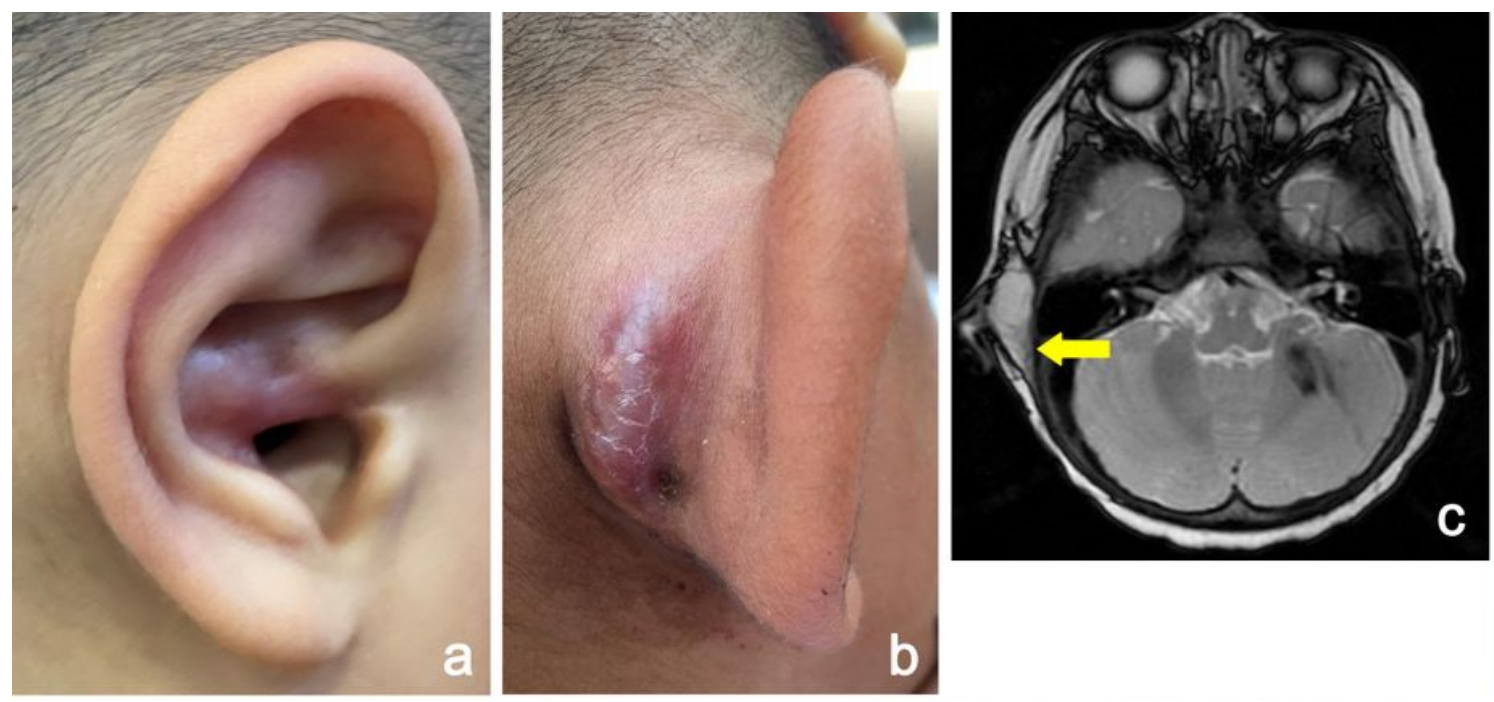

b
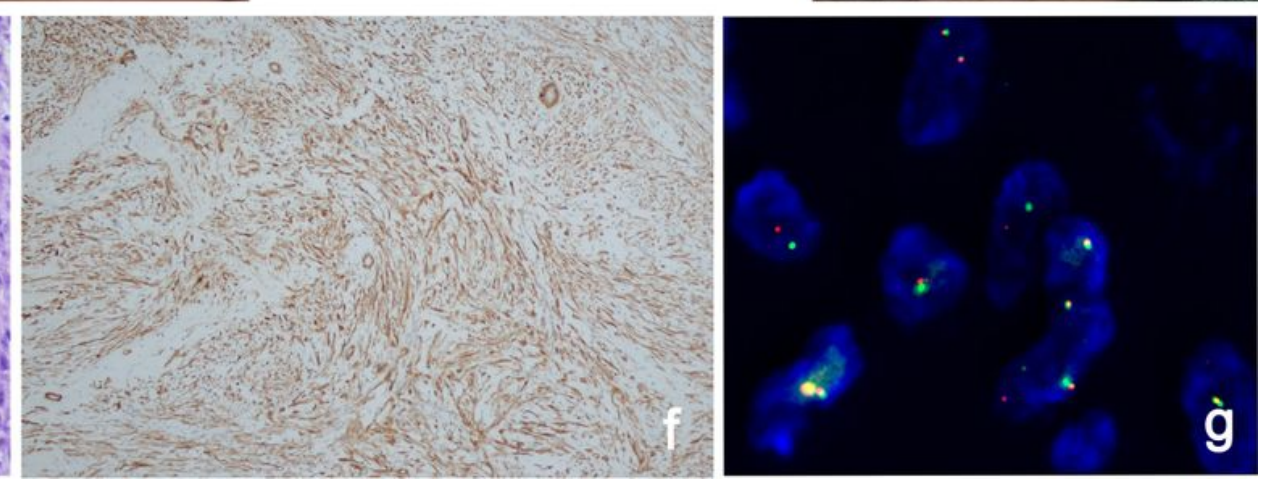

\section{Figure 2}

$a$ and $b$ are Pre-operative clinical photograph of case 2 which shows a mass in cavum conchae and back of the auricle. Fig2-c shows Axial T2WI scan of case 2 with a high signal intensity. We can find the "fascial tail" sign (arrow). Fig2-d is a photograph of ear five months after operation with an intact ear appearance. The histological changes of NF of case 2 (Fig2-e: H\&E stain, $\times 200$ ). IHC staining showed positivity for SMA (Fig2-f). FISH showing rearrangements of USP6 locus (separation of green and red signals) in case 2 (Fig2-g) of NF 

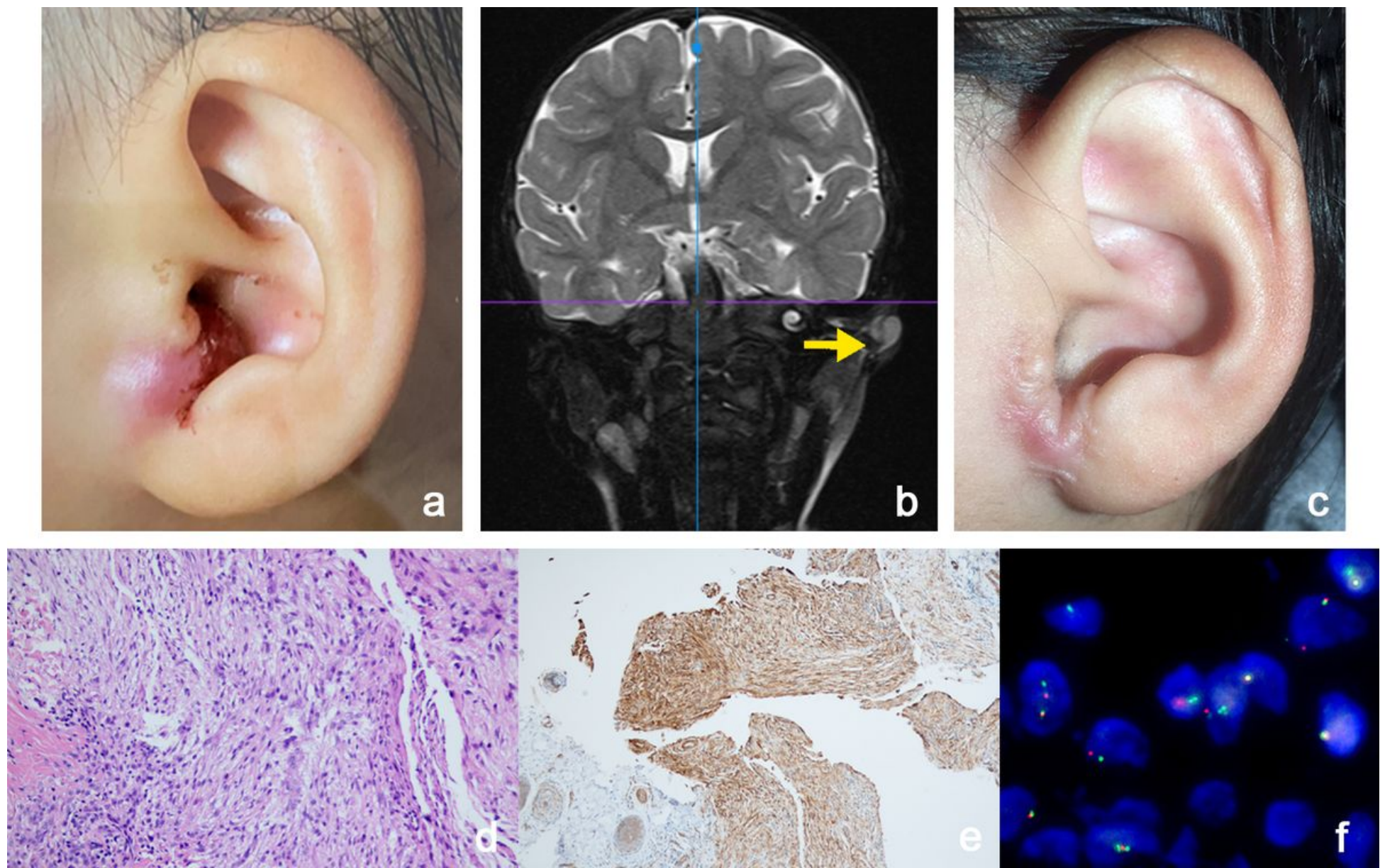

Figure 3

a is Pre-operative clinical photograph of case 3. Fig3-b show coronal T2WI of case 3. We can also find the "fascial tail" sign (arrow). Fig3-c is a photograph of ear eight months after operation with a pale pink postoperative scar. The histological changes of the lesion (Fig3-d: H\&E stain, $\times 200$ ). IHC staining showed positive for SMA (Fig3-e). FISH showing rearrangements of USP6 locus (separation of green and red signals) in case 3 (Fig3-f) 